\title{
Joint interpretation of Radiomagnetotelluric and CVES measurements in the Grundfør area, Denmark.
}

\author{
Mette Kathrine Lauridsen and Niels B. Christensen \\ Department of Earth Sciences, Aarhus University \\ Finlandsgade 8, DK-8200 Aarhus N, Denmark
}

\section{INTRODUCTION}

The radiomagnetotelluric (RMT) method has been used to collect data in the Grundfør area in Denmark to estimate the potential of the method under Danish conditions. The Grundfør area was chosen because it has been intensively mapped with several geophysical methods in connection with the Danish Environmental Research Programme. The results of previous measurements thus form a good basis for comparison.

The RMT method utilizes the electromagnetic fields from distant radio transmitters as a source and measures orthogonal horizontal electric and magnetic field pairs: $\left[E_{x}, H_{y}\right]$ or $\left[E_{y}, H_{x}\right]$ for each frequency. In Europe, where radio transmitters in the interesting frequency range are abundant, the RMT method is expected to have a large potential, and much research is presently done with the method.

\section{THE MEASUREMENTS AND THE INTERPRETATIONS}

In the Grundfør area 21 lines in the NS direction with an interline spacing of $10 \mathrm{~m}$ were measured with an interval of $5 \mathrm{~m}$ on the lines. Both pairs of fields were measured: one with the electric field parallel to the line (called NS data) and one with the electric field perpendicular to the line (EW data).

The equipment used is from the University of Cologne, Germany, and it measures orthogonal horizontal electric and magnetic field pairs. The electric field is measured with a 5 meter dipole and the magnetic field with a vertical coil. The equipment has four frequencies which are set for each polarization.

The frequencies used for the NS data were: $16.8 \mathrm{kHz}, 53.0 \mathrm{kHz}, 126.8 \mathrm{kHz} / 128.0 \mathrm{kHz}$ and 183.0 $\mathrm{kHz} / 216.0 \mathrm{kHz}$. And for the EW data: $24.0 \mathrm{kHz} / 21.4 \mathrm{kHz}, 52.0 \mathrm{kHz}, 126.1 \mathrm{kHz}$ and $225.0 \mathrm{kHz}$. They were chosen to cover the range from $10 \mathrm{kHz}$ to $250 \mathrm{kHz}$. When the reception of a certain transmitter failed a neighboring frequency was chosen.

The quality of the data is only fair. The measurements are done by fine-tuning with two knobs finding an intensity minimum of an indicator tone in a set of earphones. The fine-tuning is often ambiguous and because of the selective character of human hearing the quality deteriorates during the day.

Data have been interpreted with different one-dimensional (1D) models: two- and three-layer models with free layer boundaries, and three-, four-, and five-layer models with fixed layer boundaries. The fixed layer boundaries have been found by using the Fréchet kernel for the RMT method so that the information in data is equally distributed over the layers.

The free layer boundary models tumed out to be of no use, while the fixed layer boundary models gave good and rather similar results. The four-layer model proved optimal, as the three-layer model was too coarse and the five-layer model did not enhance the resolution. Data were interpreted using both the $L_{1}$-norm and the $L_{2}$-norm and, as expected, the $L_{1}$-norm was optimal because of its robustness to outliers in data. All model sections presented have been made using the $\mathrm{L}_{1}$-norm. 
RMT NS, five-layer model with fixed layer boundaries
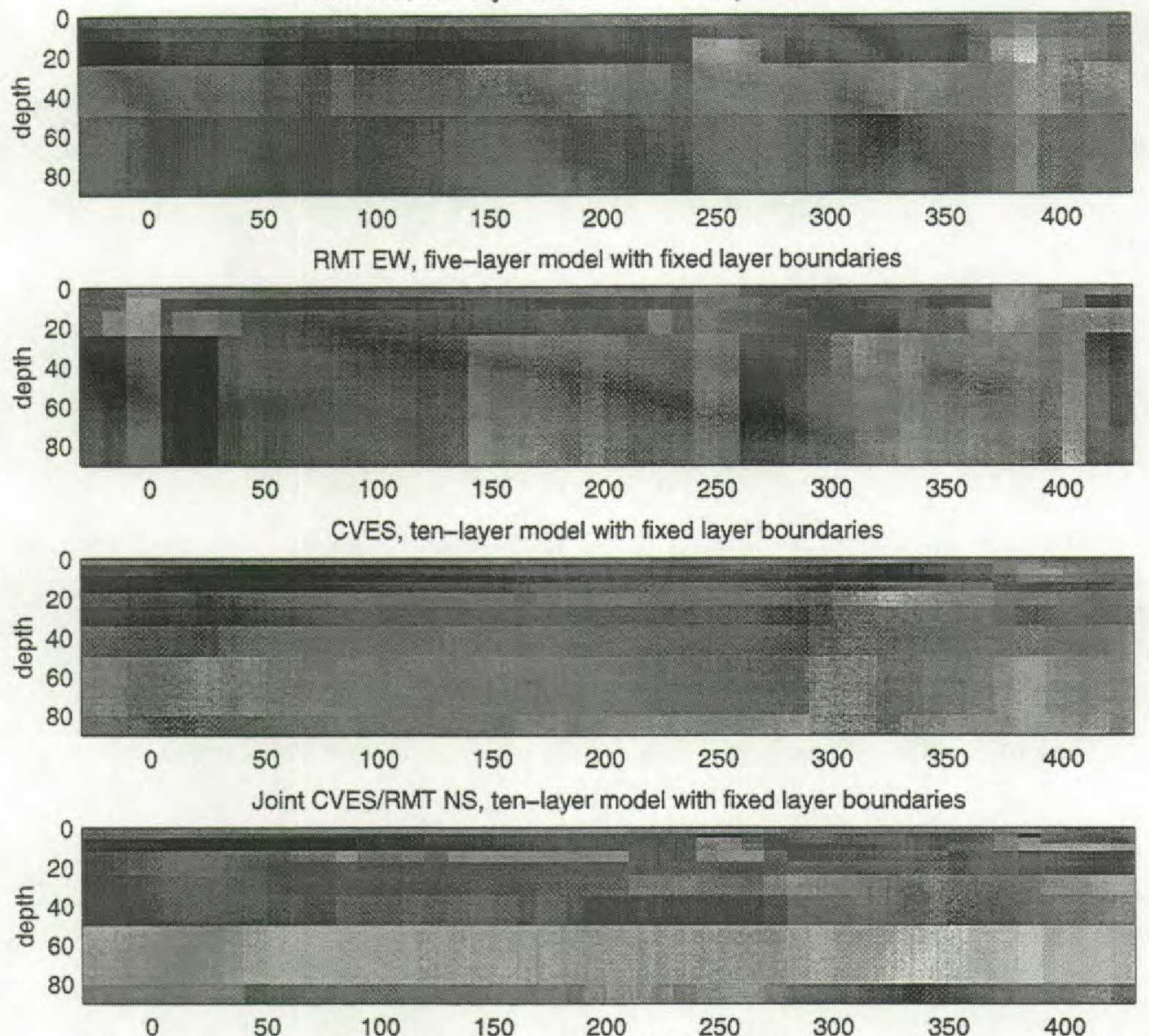

Joint CVES/RMT EW, ten-layer model with fixed layer boundaries

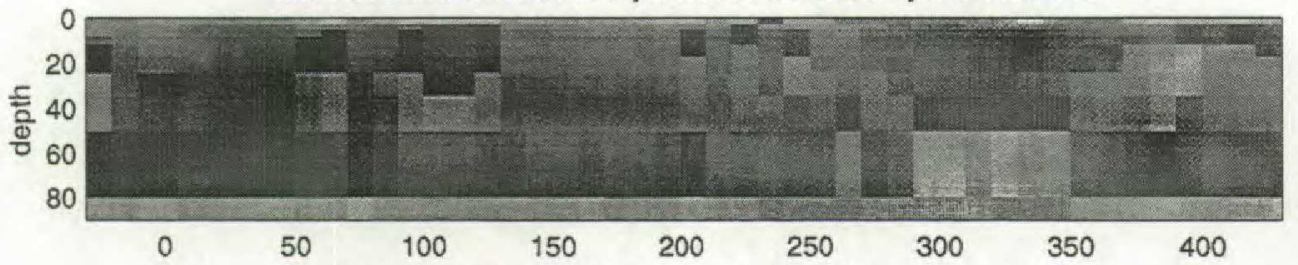

Joint CVES/RMT NS, four-layer model with free layer boundaries

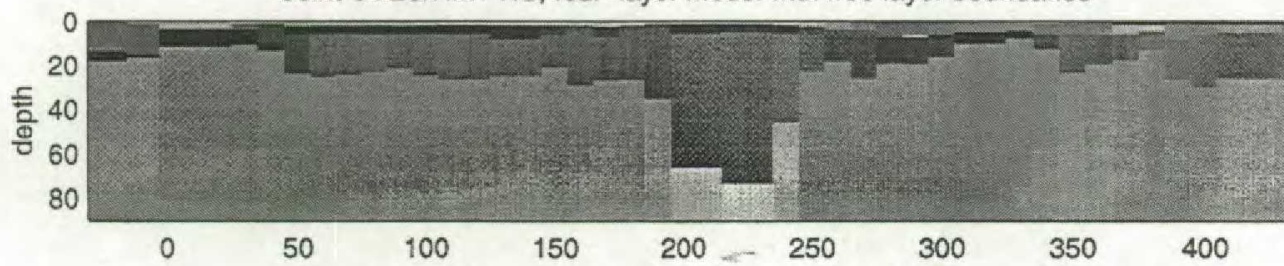

Joint CVES/RMT EW, four-layer model with free layer boundaries

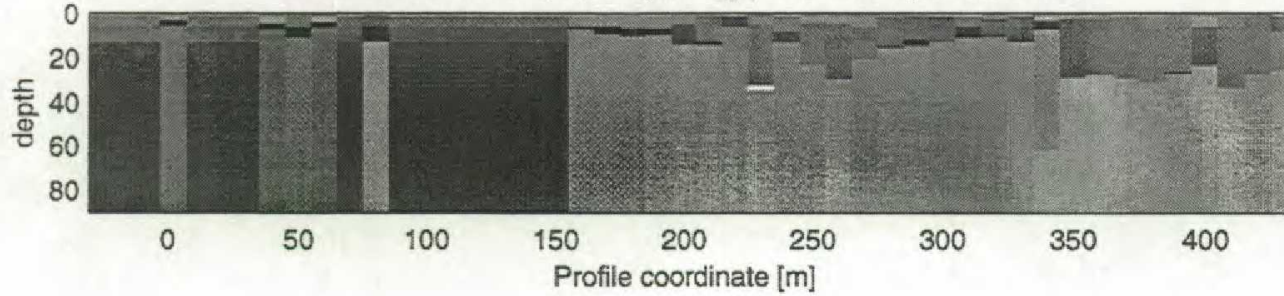

Figure 1: Model sections from interpretation of RMT and CVES data on the $+50 \mathrm{~m}$ profile line. Gray scale is logarithmic with white corresponding to $10 \Omega \mathrm{m}$ and black to $500 \Omega \mathrm{m}$. 

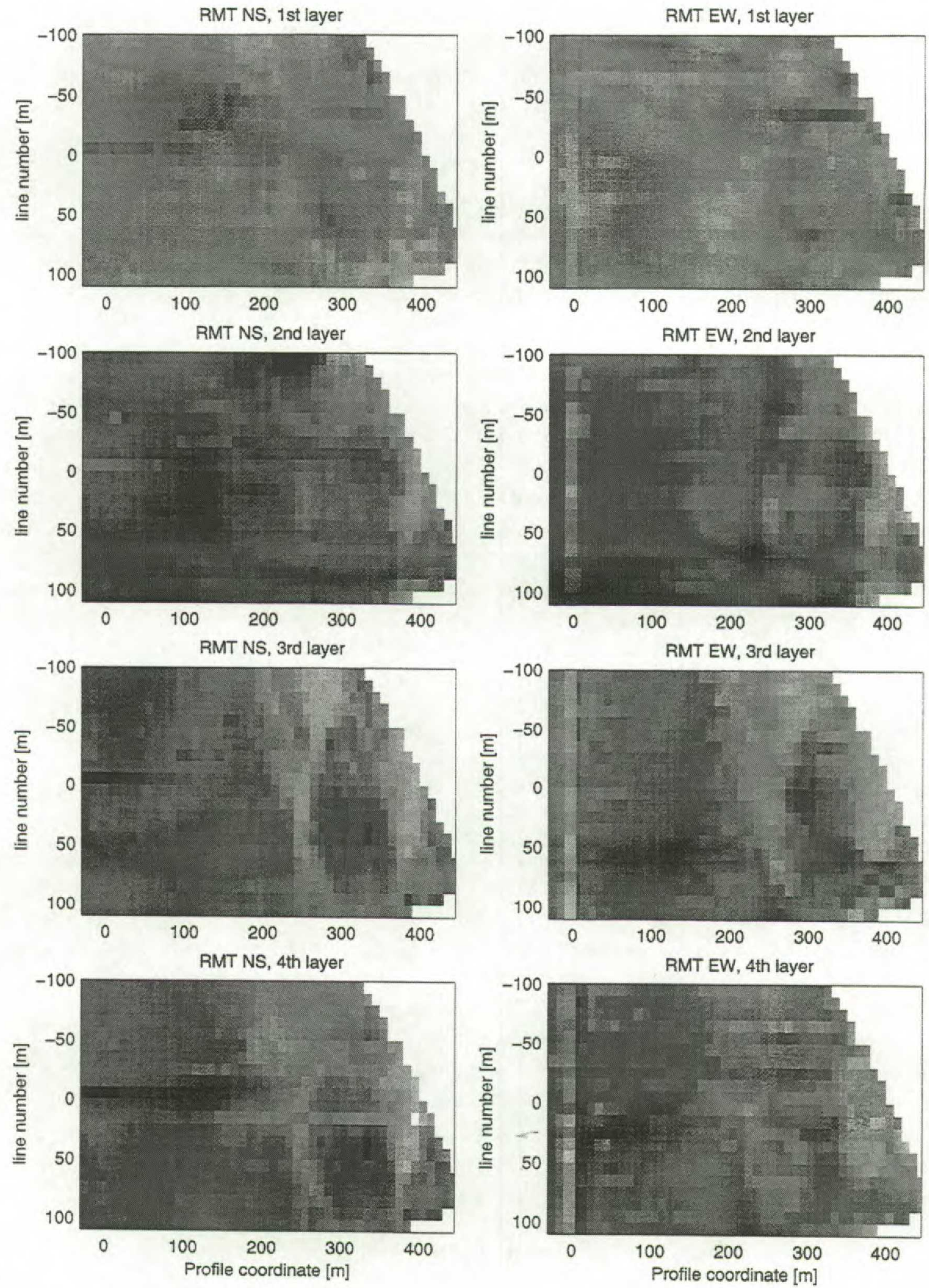

Figure 2: Contoured maps of the layer resistivities of four-layer models with fixed layer boundaries. Left column is for the NS polarization, right column is for the EW polarization. The layer boundaries are at 1.9, 6.6, and $17.1 \mathrm{~m}$ depth. Gray scale same as for Figure 1 .

Previously the area was mapped with the CVES method (Continuos Vertical Electromagnetic Sounding) on five profile lines and the RMT measurements have been made on and between these lines. The CVES data have been interpreted with a 1D ten-layer model with fixed layer boundaries. 
Using the same ten-layer model and also a four-layer model with free layer boundaries a joint interpretation between CVES data and RMT data has been done.

The results are presented as model sections of concatenated 1D models for line $+50 \mathrm{~m}$ (the longest of the CVES lines) and as horizontal depth slices of the RMT interpretations for the whole area.

\section{RESULTS}

In Figure 1 the RMT NS interpretation shows a high resistivity layer with an overburden of lower resistivity, and a high resistivity body around $320 \mathrm{~m}$ surrounded by lower resistivity. The same features are seen in the RMT EW interpretation but the section is more irregular. To facilitate comparison both interpretations have been made with a five-layer model resulting from joining layers of the ten-layer model used for the CVES data. The CVES section shows the same features as the RMT sections, although the body around $320 \mathrm{~m}$ is of a smaller vertical extent. The deeper layers have a lower resistivity than the corresponding ones in the RMT sections. The joint CVES and RMT NS interpretation appears as a mix of the two separate sections although the ninth layer has a lower resistivity than in either of the sections alone. The joint CVES and RMT EW interpretation is very irregular but the body around $320 \mathrm{~m}$ can still be seen.

The joint CVES and RMT NS interpretation with the four-layer model with free layer boundaries gives nearly the same result as the ten-layer interpretation. The joint CVES and RMT EW interpretation is as irregular as the ten layer interpretation, but the body around $320 \mathrm{~m}$ is still seen.

The results of the RMT interpretations for the whole area with four-layer models with fixed layer boundaries are presented in Figure 2 as contoured maps of the layer resistivities, corresponding to horizontal depth slices through the model for both polarizations. The upper layer is uniformly of low resistivity. The second layer has a higher resistivity, but the NS polarization has a lower resistive body which appears as just spots in the EW data. Both polarizations have a high resistivity body surrounded by lower resistivity in the third layer. This body also appears in the fourth layer for the NS polarization data, but in the EW data it is almost gone.

\section{CONCLUSION}

The quality of the RMT data recorded at Grundfør leaves much to be wished for, but with a modern multi-frequency tensor system the RMT method will probably be very useful for near-surface investigations.

The fair quality of data is probably the reason why models with fixed layer boundaries proved superior to models with free layer boundaries. The results of the interpretations of the different polarizations were different due to the multi-dimensional character of the geology.

The results of the interpretations of the RMT data were similar to the results from the CVES profiles where comparisons could be made. Joint interpretations gave reasonable results only for that RMT polarization where the electric field is measured in the same direction as that of the CVES data.

A 2D interpretation of the RMT data would probably be superior to the $1 \mathrm{D}$ interpretations presented here, but the small number of frequencies in this investigation and the fair data quality would impair the reliability of the result.

\section{REFERENCES}

Christensen, N.B., and Auken, E., 1992: SELMA - Simultaneous electromagnetic layered modeling and analysis. In: Jacobsen, B.H. (ed.): Proceedings of Interdisciplinary Inversion Workshop 1 Aarhus 1992, Methodology and Applications within Geophysics, Astronomy, and Geodesy. GeoSkrifter, Aarhus University, 41, 49-56.

Vozoff, K., 1991: The Magnetotelluric Method, in: M.N. Nabighian, ed., Electromagnetic Methods in Applied Geophysics. Society of Exploration Geophysicists.

Whittall, K.P. and Oldenburg, D.W., 1992: Inversion of Magnetotelluric Data for a OneDimensional Conductivity. Society of Exploration Geophysicists. 